\title{
Früherkennung von Strukturschäden mittels geführter Lamb-Wellen
}

\author{
Dr.-Ing. Jens Prager, Dipl.-Inf. Enrico Köppe, Dr.-Ing. Matthias Bartholmai
}

BAM Bundesanstalt für Materialforschung und -prüfung

Fachbereich 8.1 Sensorik; mess- und prüftechnische Verfahren; Unter den Eichen 87, 12205 Berlin

Email: enrico.koeppe@bam.de

Email: matthias.bartholmai@bam.de

Phone: +49 3081041914 / 1912

Fax: +49 3081041917

\section{Zusammenfassung}

Eine Reihe von Prüfproblemen in der chemischen Industrie, in Kraftwerksanlagen und in Transportsystemen ist aufgrund schwieriger Zugangsmöglichkeiten nur begrenzt technisch bzw. wirtschaftlich effizient durchführbar. Das hier vorgestellte Projekt beinhaltet die Entwicklung eines Verfahrens der zerstörungsfreien Prüfung über weite Entfernungen (bis zu 100 m), das zum Einsatz in automatisierten Messsystemen bzw. zur Integration in Langzeitüberwachungssysteme geeignet ist. Grundlage des Verfahrens ist die gezielte Anregung und Auswertung von Lamb-Wellenmoden, wodurch eine effektive Unterscheidung von Fehler- und Formanzeigen sowie eine Klassifizierung unterschiedlicher Fehlertypen möglich werden.

Der Vorteil der Nutzung geführter Wellen für die zerstörungsfreie Prüfung liegt in ihrer vergleichsweise guten Ausbreitungsfähigkeit über weite Entfernungen. Die sich daraus ergebenden Möglichkeiten der Untersuchung großer bzw. schwer zugänglicher Bereiche einer Struktur bieten innovative Ansätze für die Entwicklung robuster Structural-Health-Monitoring-Systeme (SHM). Erst durch die Verfügbarkeit leistungsfähiger Rechentechnik wird ein breiter Einsatz derartiger Verfahren möglich. Die Technik kann zur Materialcharakterisierung an ebenen bzw. zylindrischen, dünnwandigen Flächen aus Metall oder auch CFK genutzt werden. Adressierte Anwendungsfelder sind z.B. die Untersuchung von Flugzeugtragflächen oder Pipelines. Des Weiteren wird der Einsatz solcher Verfahren zur Überwachung sicherheitsrelevanter Bauteile z.B. aus Laminaten angestrebt, wie sie verstärkt in der Luft- und Raumfahrt Anwendung finden.

Im Rahmen des Projekts wurde auf Grundlage vorhandener Technik (Wellenanregung mittels UltraschallGruppenstrahlprüfköpfen) ein umfassendes Verständnis für die Technologie und anwendungsrelevante Parameter (Probenmaterial und -dimensionen, Welleneigenschaften, Anregungs-, Transmissions-, Reflektionscharakte-ristika) gewonnen und Algorithmen zur Erkennung von Schädigungsbildern (z.B. Riss, Loch, Verdünnung) entwickelt.

Neben der Algorithmusentwicklung zur Detektion und Analyse von Schadstellen, lag ein Projektschwerpunkt in der Entwicklung einer geeigneten Gerätetechnik. Als sensorisches Elemente wurde Ultraschall-Gruppenstrahlertechnik eingesetzt. Besondere Anforderungen werden an die Technik zur Anregung der Wellenmoden unter Verwendung geeigneter Piezoaktoren gestellt. In diesem Bereich existiert bislang keine kommerziell erhältliche Gerätelösung, weshalb eine Neuentwicklug der Anregetechnik realisiert wurde. Speziell für die hohe Anzahl an Aktorkanälen, die benötigt wird, um unterschiedliche Moden und Wellenformen anzuregen, wurde eine äußerst leistungsfähige Anregeeinheit entwickelt.

\section{Einleitung}

Für die Prüfung räumlich ausgedehnter Strukturen erlangten Prüfverfahren mit geführten Ultraschallwellen (guided waves) in den vergangenen Jahren zunehmend an Bedeutung. Geführte 
Ultraschallwellen breiten sich in einem Bauteil nahezu ungedämpft über weite Entfernungen aus.

Dadurch wird es möglich, von einer Beobachtungsposition - also einer Sensorposition ausgehend - weite Bereiche der Struktur zu untersuchen, wodurch sich der Aufwand für die Prüfung sowohl hinsichtlich der reinen Messzeit als auch hinsichtlich der Anforderungen an die Zugänglichkeit des Bauteils deutlich reduziert. Das Haupteinsatzgebiet des Prüfverfahrens ist gegenwärtig die Rohrleitungsprüfung. Darüber hinaus existieren vielversprechende Ansätze z.B. für die Überwachung stark beanspruchter Bauteile in der Luftfahrt.

Bei der Rohrleitungsprüfung werden Systeme eingesetzt, die es ermöglichen abhängig von den Prüfbedingungen Leitungsabschnitte bis zu $50 \mathrm{~m}$ und darüber hinaus in beide Richtungen von einem Sensorort aus zu überprüfen. Derzeit werden solche Systeme hauptsächlich zur wiederkehrenden Prüfung von Gas- und Ölpipelines eingesetzt. Insbesondere bei erdverlegten Leitungen, an Straßendurchführungen aber auch bei isolierten Rohrleitungen in Industrieanlagen treten die Vorteile einer Prüfung mit geführten Wellen deutlich zu Tage. Beim Einsatz des Verfahrens muss allerdings berücksichtigt werden, dass es sich hierbei vorrangig um ein Suchverfahren und nicht um ein Analyseverfahren handelt. Zwar ist es möglich, Veränderungen im zu prüfenden Bauteil aufzuspüren, aufgrund der physikalischen Randbedingungen ist jedoch eine exakte Orts- bzw. Positionsbestimmung oder eine Bestimmung von Fehlerart und -größe mit dem derzeitigen Stand der Technik nicht in dem Maße möglich, wie es die klassische Ultraschallprüfung zulässt. Die Fehlerauffindewahrscheinlichkeit insbesondere bei kleinen Fehlern ist verglichen mit den klassischen Verfahren etwas geringer.

In der Luftfahrttechnik wird in zunehmendem Maße versucht, geführte Wellen zur Prüfung hoch beanspruchter Bauteile einzusetzen [1]. Insbesondere der Einsatz neuer Werkstoffe, wie faserverstärkter Kunststoffe (CFG, GFK), die andere Schädigungsmechanismen als herkömmliche metallische Werkstoffe aufweisen, verlangt nach geeigneten neuen Prüftechniken. Mittels geführter Ultraschallwellen, die sich in weiten Bereichen der Außenhaut des Rumpfes oder der Tragflächen ausbreiten, kann der Zustand dieser Bauteile effektiv analysiert werden. Es ist möglich, geeignete Aktor-Sensor-Kombinationen in die Faserverbundwerkstoffe einzulaminieren, die mit einem fest installierten Online-Überwachungssystem ausgewertet werden.

Ihr volles Potenzial kann eine Prüftechnik mit geführten Wellen erst erreichen, wenn sie im Rahmen einer Strukturüberwachung (SHM) zur Schadensfrüherkennung eingesetzt wird. Hierbei geht es nicht mehr vorrangig darum, in einem einmaligen Prüfvorgang Fehler exakt zu lokalisieren und zu charakterisieren, vielmehr wird nur die Integrität des Bauteils durch sequentielle Prüfläufe überwacht. Mit dieser Überwachung in regelmäßigen Abständen, die günstigenfalls mit einer ortsfesten Sensoranordnung durchgeführt wird, können aus der Historie der Messreihen Aussagen über Veränderungen am Bauteil abgeleitet werden. Dieser Ansatz ist deutlich empfindlicher, und es können selbst kleinste Veränderungen aufgespürt werden. Durch die Definition geeigneter Kriterien wird es außerdem möglich, die Lebensdauer der Bauteile möglichst vollständig auszunutzen bzw. Aussagen über die verbleibende Restlebensdauer abzuleiten.

Im Gegensatz zur klassischen Ultraschallprüfung, bei der hauptsächlich Longitudinal- oder Transversalwellen angeregt werden, können sich bei der Anregung geführter Wellen verschiedene Wellenmoden ausbilden. Bis auf wenige Ausnahmen ist die Ausbreitung dieser Wellenmoden dispersiv, d.h., die Schallausbreitungsgeschwindigkeit ist frequenzabhängig. Die Dispersion erschwert die Auswertung der Messdaten und erfordert den Einsatz qualifizierter Auswertealgorithmen. Durch Auswahl geeigneter schmalbandiger oder modenselektiver Anregeverfahren kann der Einfluss der Dispersion zwar minimiert, jedoch nicht beseitigt werden. Die Entwicklung geeigneter Anrege- und Sensortechnik ist von entscheidender Bedeutung.

\section{Physikalische Grundlagen}

Während in homogenen dreidimensional unendlich bzw. halbunendlich ausgedehnten Medien die Schallausbreitung hauptsächlich durch reine Longitudinal- und Transversalwellen erfolgt, mit einer Teilchenauslenkung entweder in Ausbreitungsrichtung oder senkrecht dazu, findet die Schallausbreitung in räumlich begrenzten, nur ein- oder zweidimensional unendlich ausgedehnten Körpern (Stäben, Rohren, Platten usw.) in Form von geführten Wellen statt. Die einfachste Form einer geführten Welle tritt 
in Plattenstrukturen auf. Entlang der Platte breiten sich Plattenwellen (Lamb-Wellen) aus. Dabei werden symmetrische und asymmetrische Plattenwellen unterschieden. In Bild 1 sind die Teilchenauslenkungen einer asymmetrischen und einer symmetrischen Mode 0. Ordnung (AO und S0) dargestellt. Es ist ersichtlich, dass eine Teilchenbewegung in beide Raumrichtungen gleichzeitig stattfindet, also sowohl in Wellenausbreitungsrichtung als auch senkrecht dazu. Das ist an allen Punkten, abgesehen von den „,neutralen Fasern“ der Fall. Im Gegensatz zu den Moden höherer Ordnung besitzen die Moden 0 . Ordnung keine Cut-Off-Frequenz, unterhalb derer sie nicht mehr ausbreitungsfähig sind. Die SO-Mode wird in der klassischen Akustik auch als Quasi-Longitudinalwelle bezeichnet. Die A0-Mode ist die Biegewelle der Platte. Bei höheren Moden ist die Verteilung der Teilchenauslenkung über dem Querschnitt komplexer. Mit zunehmender Ordnung nimmt die Anzahl der „neutralen Fasern“ zu, in denen die Teilchenbewegung in einer Richtung in Ruhe ist. Es muss berücksichtigt werden, dass die Verteilung der Teilchenauslenkung über den Querschnitt in jedem Fall auch frequenzabhängig ist [2].
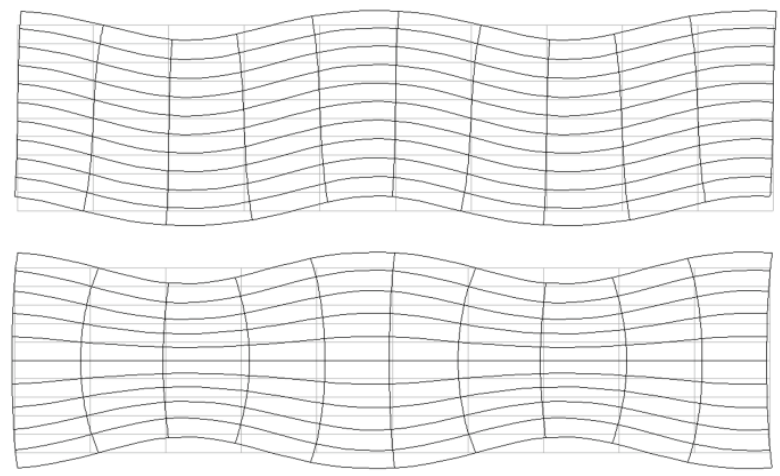

Bild 1: Teilchenauslenkung der asymmetrischen (oben) und symmetrischen (unten) Plattenwelle (LambMode) 0. Ordnung.

Eine Sonderform der Plattenwellen sind die SH-Wellen, horizontal polarisierte Wellenmoden, deren Teilchenauslenkung senkrecht zur Ausbreitungsrichtung, aber in der Plattenebene erfolgt. Die 0. Ordnung der SH-Moden (SHO) ist als einzige nichtdispersiv. Sie spielt aber wegen ihrer schlechten Anregbarkeit für die Ultraschallprüfung nur eine untergeordnete Rolle. In Bild 2 sind beispielhaft die Dispersionskurven für eine Aluminiumplatte dargestellt. Bemerkenswert ist, dass die x-Achse der Kurven das Produkt aus Frequenz und Plattendicke $(f \cdot d)$ darstellt, woraus sich eine einfache Skalierbarkeit der Prüfprobleme ergibt. Die Kurven zeigen sowohl die Phasen- als auch die Gruppengeschwindigkeiten. Während sich aus der Phasengeschwindigkeit die Wellenlänge der Schallwelle bestimmen lässt, gibt die Gruppengeschwindigkeit die Laufzeit der spektralen Signalbestandteile in Abhängigkeit ihrer Frequenz an.

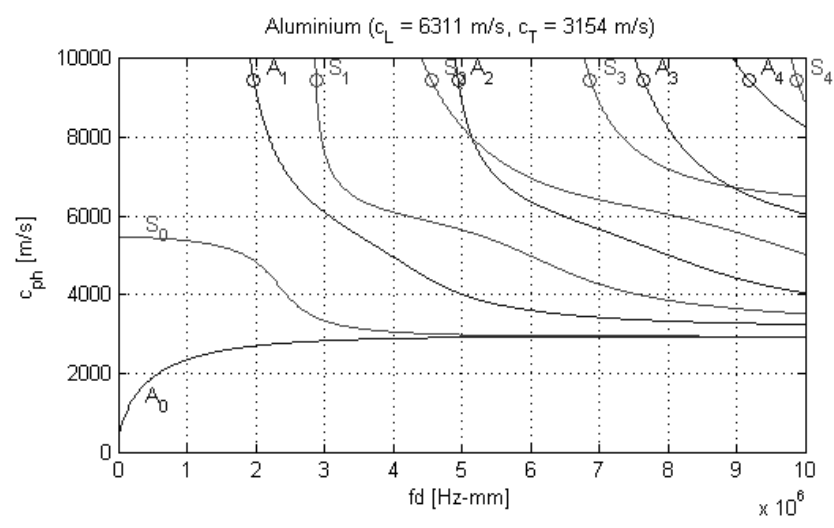




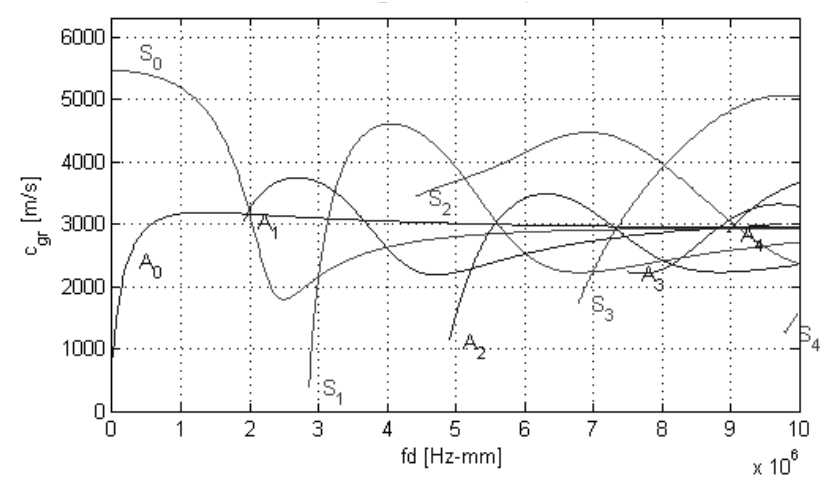

Bild 2: Dispersionskurven für eine Aluminiumplatte der Dicke d. Phasengeschwindigkeiten (oben) und Gruppengeschwindigkeiten (unten) der asymmetrischen (Ax) und symmetrischen (Sx) Moden.

Um die Wellenausbreitung in Rohren zu verstehen, kann man sich das Rohr als gerollte Platte vorstellen. Teilt man nun die ausbreitungsfähigen Wellen in eine axiale und eine Umfangskomponente, wird sichtbar, dass für die letztgenannte Komponente nur diskrete Wellenzahlen in Frage kommen, die einen geschlossenen Wellenzug auf dem Umfang bilden können. Für die Teilchenauslenkungsverteilung über die Wanddicke trifft wiederum das bereits über Platten gesagte zu. Unter Berücksichtigung dieser Bedingungen ergeben sich drei mögliche Typen von Wellenmoden: Biegemoden, Longitudinalmoden und Torsionsmoden. Die Torsionsmoden leiten sich von den SH-Plattenmoden ab. Auch hier ist die Grundmode $\mathrm{T}(0,1)$ als einzige dipersionsfrei. Für die Wellenausbreitung in Rohren lassen sich ähnliche Dispersionsdiagramme wie für Platten erstellen.

Bei der Ultraschallprüfung wird im Allgemeinen die Summe aus der Laufzeit eines kurzen Schallimpulses vom Sender zum Fehler und der Laufzeit des am Fehler reflektierten Signals zum Empfänger für die Lokalisation der Fehlerposition verwendet. Meist sind Sender und Empfänger der gleiche Sensor, wodurch in der klassischen Ultraschallprüfung der Impuls die gleiche Wegstrecke zweimal mit gleicher Geschwindigkeit durchläuft. Für die Prüfung mit geführten Wellen sind die Zusammenhänge etwas komplexer, zum einen wegen der Breitbandigkeit der kurzen Sendeimpulse, zum anderen wegen der auftretenden Modenumwandlung.

Breitbandige Impulse „zerfließen“ aufgrund der starken Dispersivität, die bei der Ausbreitung geführter Wellen auftritt. Die Folge ist, dass die einzelnen Frequenzbestandteile des Impulses zu unterschiedlichen Zeiten am Empfänger ankommen, und es ist sehr schwierig, aus der Laufzeit die Position des reflektierenden Fehlers zu bestimmen. Durch das „Zerfließen“ des Impulses sinkt außerdem seine maximale Amplitude, was sich negativ auf das Signal-Rausch-Verhältnis auswirkt.

Für eine beliebige Anregung entstehen in der Struktur - unter Berücksichtigung des Spektrums des Anregesignals und der Cut-Off-Frequenzen der einzelnen Moden - alle ausbreitungsfähigen Moden. Treffen die Moden während ihrer Ausbreitung auf Inhomogenitäten in der Struktur - das können Geometrieänderungen, Werkstoffänderungen aber auch Fehlstellen, wie Risse oder Querschnittsänderungen durch Materialabtrag (z.B. Korrosionsschäden) sein - werden die Moden reflektiert, und es kann zusätzlich Modenumwandlung auftreten. D.h., abhängig von der Geometrie der Inhomogenität, der einfallenden Mode und der Frequenz findet die Reflexion nicht nur in der eingeschallten Mode statt, sondern es werden ebenfalls ausbreitungsfähige, neue Moden erzeugt. Es ist zu erwarten, dass das Echosignal deutlich komplexer ist als das einfallende. Selbst wenn nur eine Mode vom Sender angeregt wird, können im Echosignal mehrere Moden enthalten sein, die unterschiedliche Ausbreitungsgeschwindigkeiten besitzen. Ziel der Entwicklung eines Prüfverfahrens ist es daher, eine geeignete monomodale Anregung zu realisieren und Auswertestrategien zu implementieren, die alle wichtigen Informationen aus dem Empfangssignal extrahieren können.

\section{Aktorik und Sensorik}

Wie schon erwähnt ist es für die Entwicklung eines effizienten Prüfverfahrens von besonderer Bedeutung, einzelne Moden selektiv anregen zu können. Ziel ist es, eine definierte Auslenkungsverteilung auf die Bauteiloberfläche aufzuprägen. Da die Teilchenauslenkung an der Oberfläche fast immer mehrere Schwingungskomponenten enthält, ist es nachrangig, welche Bewegungskomponente angeregt wird. Neben einigen Sonderverfahren, wie z.B. Laseranregung, magnetostriktive Anregung oder Anregung mit 
Luftschall werden hauptsächlich piezoelektrische Wandler zur Generierung der Ultraschallwellen verwendet. Da der piezoelektrische Effekt reziprok ist, kann der Aktor auch gleichzeitig als Sensor zum Empfang des reflektierten Signals verwendet werden. Je nach anzuregender Bewegungsrichtung werden sowohl piezoelektrische Dickenschwinger als auch Scherschwinger eingesetzt. Zur Ankopplung des Wandlers an die Oberfläche der Struktur werden die Piezoschwinger meist angepresst oder aufgeklebt, um die Kraftübertragung zu gewährleisten. Dickenschwinger können auch mit Öl oder Wasser angekoppelt werden.

Für die selektive Anregung einzelner Moden wendet man das Prinzip der Spuranpassung an. Schaut man bei einem $f \cdot d-W e r t$ ins Dispersionsdiagramm, haben zumindest bei niedrigen Frequenzen alle Moden unterschiedliche Phasengeschwindigkeiten und damit auch unterschiedliche Wellenlängen. Ziel ist es, einen Wandler zu konstruieren, der bei Anregung mit der Frequenz f genau diese Wellenlänge auf die Oberfläche aufprägt.

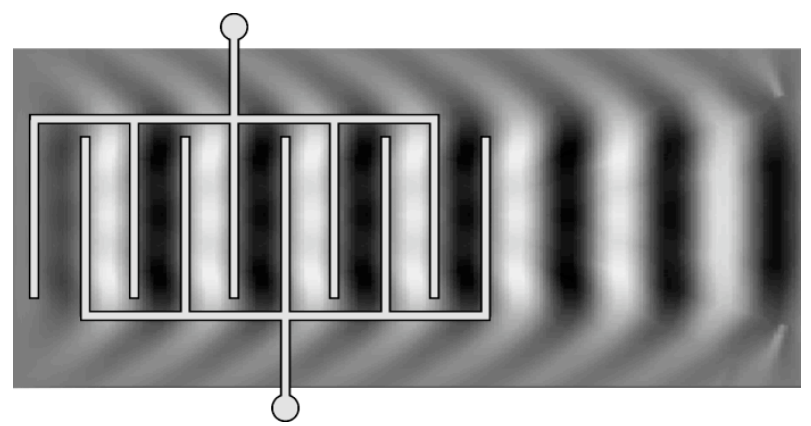

Bild 3: Anregung einer Plattenmode mit einem Interdigitalwandler (Schematische Darstellung der Elektroden und der Auslenkung der Plattenoberfläche).

Für Plattenstrukturen lässt sich dies beispielsweise mit Interdigitalwandlern realisieren. Bei diesen Wandlern, die in Bild 3 dargestellt sind, werden auf einem Trägermaterial, das mit einem piezoelektrischen Material beschichtet ist (z.B. PVDF [3], einem Kunststoff mit piezoelektrischen Eigenschaften oder PZT-Keramik [4]), zwei Elektroden mit kammartig ineinander greifender Form aufgebracht. Der Abstand der Mittellinien der einzelnen Finger muss dabei exakt der halben Spurwellenlänge entsprechen und legt die angeregte Mode fest. Erregt man nun die zwei Elektroden gegenphasig, wird eine räumliche harmonische Spannungsverteilung, die im Piezomaterial erzeugt wird, auf die Oberfläche der Struktur aufgeprägt. Wird der Transducer als Empfänger verwendet, ergibt sich ebenfalls eine wellenlängenselektive Empfangsempfindlichkeit. Interdigitalwandler werden meist auf einem flexiblen Trägermaterial gefertigt, wodurch sie sich auch an gekrümmte Bauteiloberflächen anpassen lassen. Nachteilig ist, dass der Arbeitspunkt, also die angeregte Wellenlänge des Wandlers, durch seine Geometrie festgelegt ist. Die Anregung einer Mode bei unterschiedlichen Frequenzen mit dem gleichen Wandler ist somit nicht möglich.

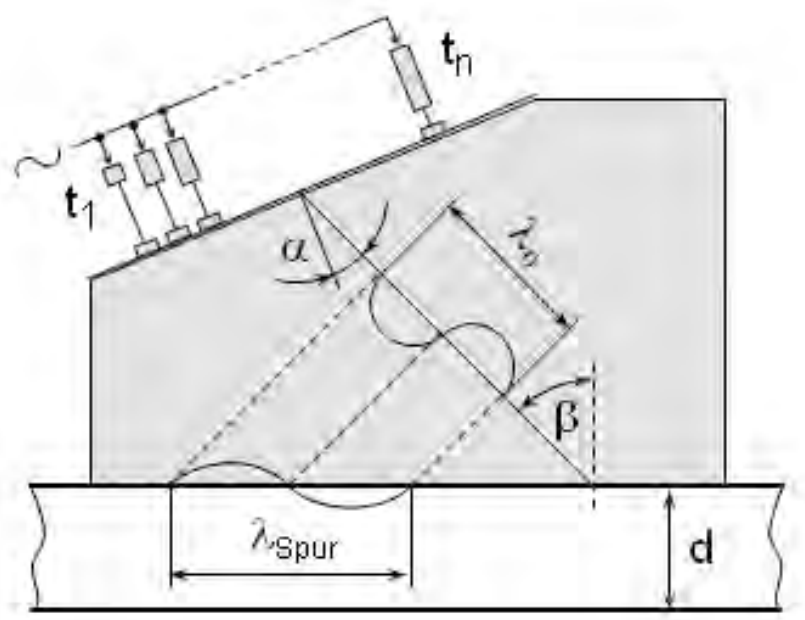

Bild 4: Anwendung eines Gruppenstrahler-Prüfkopfes zur selektiven Anregung von Wellenmoden auf Platten. 
Diese Einschränkung entfällt bei Anregemethoden, die mit Winkelprüfköpfen mit veränderlichen Keilwinkeln arbeiten. Während die Änderung des Keilwinkels früher mechanisch vorgenommen wurde, verwendet man heutzutage Gruppenstrahlerprüfköpfe bei denen der Einfallswinkel $\beta$ der Wellenfront auf die Prüfkopfsohle und damit die Spurwellenlänge elektronisch gesteuert werden kann. Das Setup ist in Bild 4 dargestellt. Bild 5 (links) zeigt das Frequenz-Wellenzahl-Spektrum der Normalkomponente des Schallfeldes auf der Prüfkopfsohle [5]. Darübergelegt sind die Dispersionskurven der Platte, diesmal in Frequenz-Wellenzahl-Darstellung. Erwartet wird, dass eine Erregung der Moden nur dort stattfindet, wo eine Dispersionskurve das Feld der Prüfkopfsohle schneidet. Bild 5 (rechts) stellt das resultierende Spektrum auf der Platte dar. Wie erwartet wird fast ausschließlich die S0-Mode angeregt. Das vorgestellte Sensorprinzip ist natürlich auch ohne Keil anwendbar, wobei die einzelnen Dickenschwinger des Sensors so angesteuert werden müssen, dass sich die erforderliche Auslenkungsverteilung auf der Oberfläche der Struktur einstellt. Die Anpassung an gekrümmte Bauteiloberflächen ist nur durch Fertigung einer angepassten Prüfkopfsohle möglich.
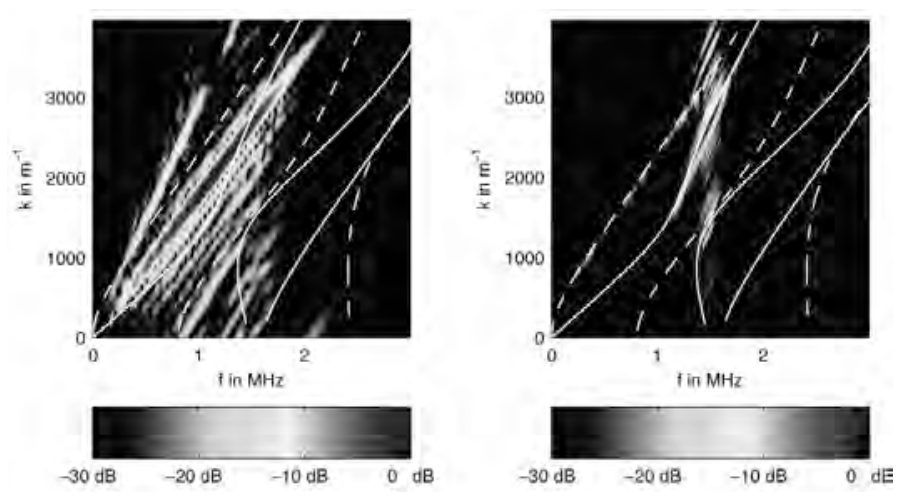

Bild 5: Selektive Anregung der S0-Mode mit einem Gruppenstrahler. Links: Frequenz-WellenzahlSpektrum der Normalkomponente der Auslenkung der Prüfkopfsohle. Rechts: Angeregtes Spektrum auf der Platte. Dispersionskurven der asymmetrischen (gestrichelt) und symmetrischen Moden (durchgezogen) sind in aufsteigender Reihenfolge.

Während die bisher beschriebenen Sensoren hauptsächlich für die Anregung geführter Wellen in Platten, Blechen oder anderen plattenähnlichen Strukturen eingesetzt werden, stehen für die Anregung von Moden in Rohrleitungen und anderen zylinderförmigen Bauteilen spezielle Sensorarrays zur Verfügung. Ein solches Sensorarray, bei dem mehrere Sensoren gleichmäßig um den Umfang des Bauteils verteilt sind, ist in Bild 6 dargestellt. In der gezeigten Konfiguration sind jeweils acht Scherschwinger in zwei Ringen angeordnet. Die Schwingrichtung der Sensoren ist senkrecht zur Längsachse des Rohres, da hier Torsionsmoden angeregt werden sollen. Insbesondere die Torsionsmode T(0,1), die sich dispersionsfrei ausbreitet, wird häufig für die Anregung der Struktur verwendet. Die Ankopplung erfolgt durch Anpressen des Sensors an die Bauteiloberfläche mittels Federkraft, so dass die Kraft durch Reibung übertragen wird. Durch die phasenverschobene Ansteuerung der einzelnen Ringe wird eine Richtwirkung erzeugt. Damit können beide Richtungen des Bauteils nacheinander untersucht werden. Um Moden höherer Ordnung anzuregen, werden die Sensoren eines Rings phasenverschoben entsprechend der Auslenkungsverteilung der anzuregenden Mode angesteuert. Gleiches gilt auch für den Empfang. Hier kann aus dem Phasenbezug der Empfangssignale der einzelnen Sensoren untereinander die einlaufende Mode identifiziert werden. Da fast alle Moden höherer Ordnung auch Schwingungskomponenten in jede mögliche Raumrichtung aufweisen, sind mit der beschriebenen Sensoranordnung auch Biege- bzw. Longitudinalmoden nachweisbar. Ggf. lässt sich die Schwingungsrichtung der Sensoren um $90^{\circ}$ drehen oder die Sensoren werden durch Dickenschwinger ersetzt. Während das dargestellte Sensorarray nur für einen spezifischen Bauteildurchmesser geeignet ist, sind auch flexiblere Konstruktionen möglich, bei denen die Sensoren auf einem Gürtel angeordnet sind, der z.B. durch Druckluft an die Bauteiloberfläche angepresst wird. 


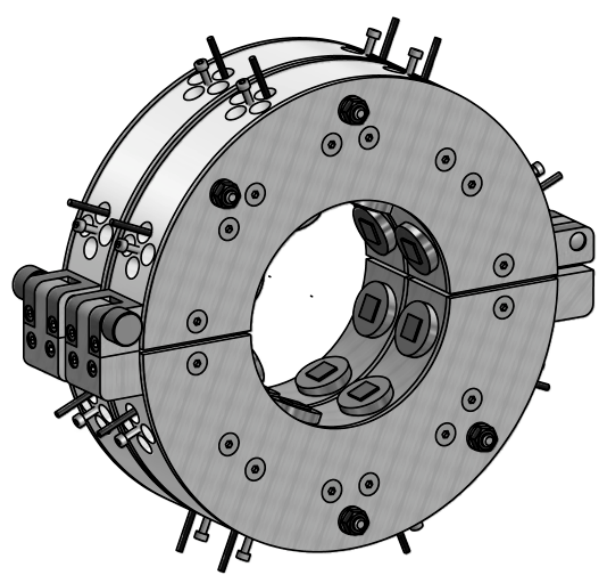

Bild 6: Sensorarray zur Erzeugung von geführten Wellen in Rohren.

\section{Signalerzeugung und -verarbeitung}

In der klassischen Ultraschallprüfung erfolgt die Anregung der Sensoren ausschließlich durch sehr kurze Dirac-ähnliche Impulse. Diese Impulse sind sehr breitbandig. Die Piezoschwinger in den Prüfköpfen werden damit bei ihrer mechanischen Resonanz angeregt. Aufgrund der mechanischen Dämpfung, die durch die Konstruktion des Kopfes bedingt ist, schwingen die Resonatoren schnell aus und erzeugen kurze, aber immer noch recht breitbandige Schallimpulse, deren Mittenfrequenz durch die Geometrie des Schwingermaterials festgelegt ist. Die kurzen Impulse sind für eine genaue Fehlerlokalisation in Tiefenrichtung entscheidend. Für die Anregung geführter Wellen ergeben sich jedoch abweichende Anforderungen: a) nach schmalbandigen Signalen, um den Einfluss der Dispersion zu minimieren und b) nach einer elektronischen Anpassung der Mittenfrequenz des Schallimpulses zur Auswahl des geeigneten Arbeitspunktes aus dem Dispersionsdiagramm. (Es sei erwähnt, dass durch das sequenzielle Prüfen eines Bauteils bei mehreren Frequenzen das Prüfergebnis deutlich verbessert werden kann.) Um diese Anforderungen zu erfüllen, erfolgt die Anregung meist mit Sinusburst-Signalen. Für die Ansteuerung der Sensoren werden dabei Spannungsamplituden $\geq 100 \mathrm{~V}$ benötigt. Dadurch wird eine geeignete Signalamplitude im Material erzeugt und ein ausreichendes Signal-Rausch-Verhältnis gewährleistet. Sowohl Sender als auch Empfänger müssen mehrkanalig aufgebaut werden, damit die Phasenverschiebung zwischen den einzelnen Sensoren erreicht werden kann, die ja Grundvoraussetzung der vorgestellten Anregeverfahren ist.

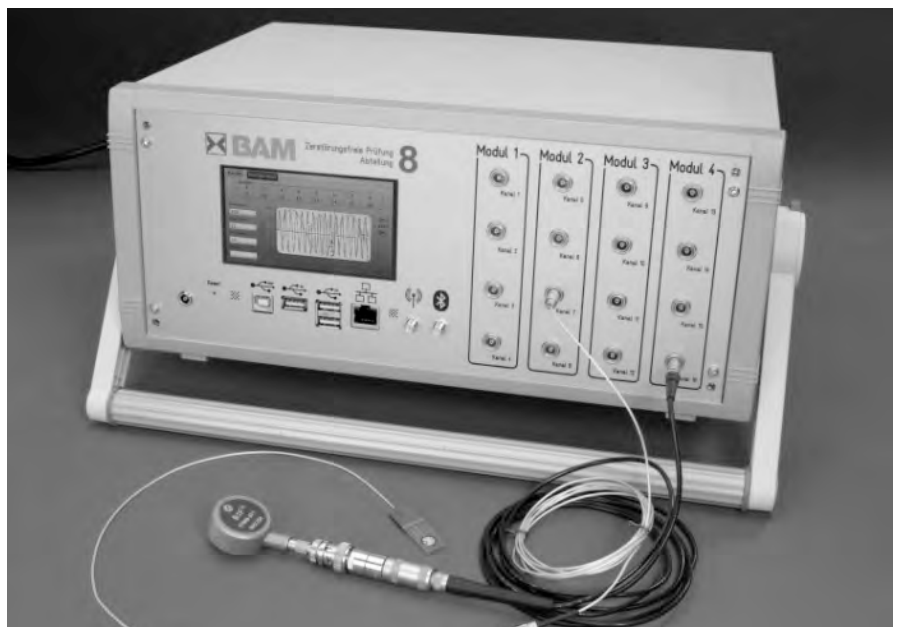

Bild 7: 16-kanalige Prüfhardware zur Anregung geführter Wellen. 


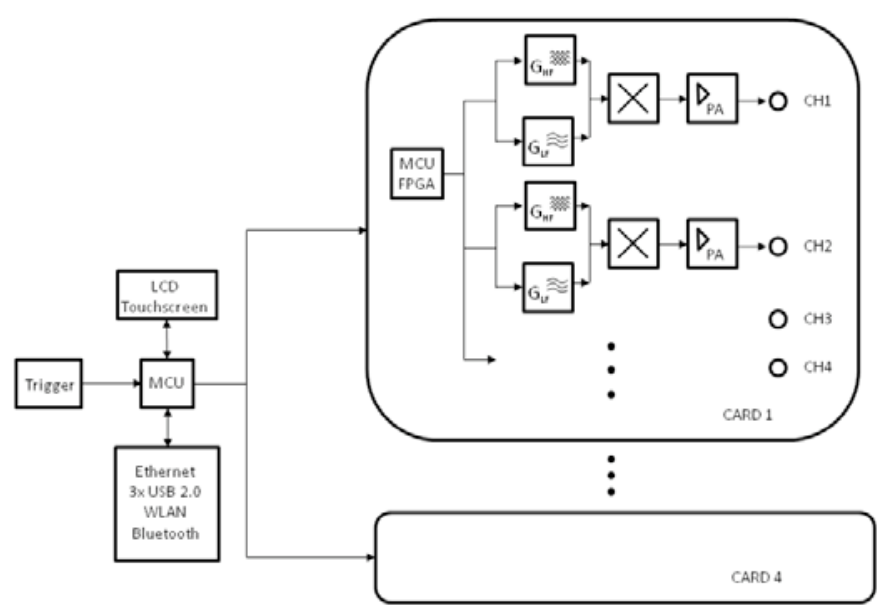

Bild 8: Blockschaltbild des Senders der Prüfhardware.

Zielsetzung des Projekts „Fehlernachweis mit geführten Wellen unter Verwendung der Gruppenstrahlertechnik“, in dem die BAM Fachbereiche „Akustische und Elektromagnetische Verfahren“ sowie „Sensorik, mess- und prüftechnische Verfahren“zusammen arbeiten, ist es eine innovative Gerätetechnik zu entwickeln, die Multikanalanregung und -erfassung geführter Lamb-Wellenmoden ermöglicht.

Bild 7 zeigt das Ultraschallprüfsystem, das speziell auf die Anforderungen der Ultraschallprüfung mit geführten Wellen zugeschnitten ist. Das Blockschaltbild des Senders ist in Bild 8 dargestellt. Die Sendestufen sind 16-kanalig ausgeführt, verteilt zu 4 Kanälen auf 4 Karten, und bestehen aus DDSSignalgeneratoren (Direct Digital Synthesis), die Sinussignale zwischen $50 \mathrm{kHz}$ und $1,5 \mathrm{MHz}$ zur Verfügung stellen. Dazu wird die Kurvenform von einer Steuer-CPU erzeugt und in die DDS-Generatoren geladen. Die Synchronisation der Generatoren erfolgt ebenfalls über die CPU, die zum einen die Phasenverschiebung der Sinussignale als auch die zeitliche Steuerung der Hüllkurven realisiert. Anschließend wird das Signal mit integrierten Leistungs-Operationsverstärkern mit hoher Slew-Rate verstärkt, um an den Piezosensoren eine Maximalamplitude von $\pm 100 \mathrm{~V}$ bereit zu stellen. Ein Ausgangssignal ist beispielhaft in Bild 9 dargestellt.
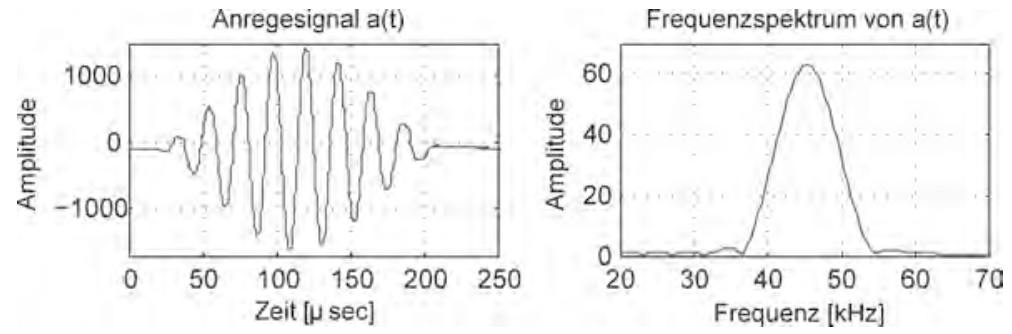

Bild 9: Anregesignal im Zeit- und Frequenzbereich

Das System aktiviert jeden einzelnen Aktor mit einer frei erzeugbaren eingehüllten Sinuskurve. bzw. Die Grund- bzw. Anregungsfrequenz ist dabei zwischen $50 \mathrm{kHz}$ und $1,5 \mathrm{MHz}$ einstellbar, bei einer wählbaren Hüllkurve von $1 \mathrm{kHz}$ bis $100 \mathrm{kHz}$. Die Anregung des Piezoaktors ist darüber hinaus über die Amplitude regelbar. Die Amplitude kann im Bereich zwischen $\pm 10 \mathrm{~V}$ bis $\pm 100 \mathrm{~V}$ bei einer Leistung von ca. $300 \mathrm{~W}$ pro Kanal eingestellt werden. Für die Generierung der Lamb-Welle kann somit jeder einzelne Anregekanal individuell und abhängig von den Eigenschaften des Piezoaktors (Referenzfrequenz oder Eigenkapazität) angepasst werden. Die Möglichkeit der Zeitsynchronität ist gewährleistet, da alle Kanäle mit der gleichen Taktquelle verbunden sind und somit ein Fehler durch eine Verschiebung bzw. Taktdifferenz von einzelnen Taktquellen ausgeschlossen wird. Ausgehend von der Zeitsynchronität kann mittels des realisierten Aufbaus und der verwendeten Hardware für jeden einzelnen Kanal eine Phasenverschiebung in Stufen von $11,25^{\circ}$ eingestellt werden. Die Phasenverschiebung von $11,25^{\circ}$ zwischen 2 Kanälen entspricht bei einer Grundfrequenz von 1,5 MHz einem Zeitversatz von 2 ns. Die Phasenverschiebung wird dabei immer relativ zum Kanal 1 festgelegt und ist nicht limitiert. Die Zeitsynchronität und die feingranulare Einstellung der Phasenverschiebung bieten die Grundlage für eine sehr genaue Erzeugung 
der gewünschten Lamb-Wellenmoden. Für jeden Kanal können die Parameter Anregefrequenz, Hüllkurvenfrequenz, Phasenverschiebung bzw. Zeitversatz relativ zu Kanal 1 und Amplitude individuell und unabhängig voneinander konfiguriert werden.

Nachdem das Sendesignal abgeklungen ist, kann mit dem Empfang begonnen werden. Die Eingangsstufe der Empfangsschaltung ist spannungsfest ausgelegt, da sie auch mit dem Sendesignal beaufschlagt wird und nach Abklingen des Sendesignals schnell mit hoher Empfindlichkeit das Empfangssignal aufzeichnen kann. Im Gegensatz zum Sender ist die Empfangsstufe einkanalig aufgebaut und wird sequentiell auf alle Kanäle einer Karte gemultiplext. Es müssen folglich 4 Messungen durchgeführt werden, um den Empfangsvektor vollständig zu füllen. Diese Betriebsweise wird durch die Zeitinvarianz der Prüfanordnung ermöglicht und stellt einen Kompromiss zwischen Geräteaufwand und Messzeit dar. Die Anbindung an einen Messrechner kann über eine USB-, LAN- oder WLAN-Verbindung erfolgen, die sowohl die Steuerdaten als auch die Messwerte überträgt. Die Firmware basiert auf einem Linux-Kernel.

\section{Ausblick}

Der Einsatz geführter Wellen für die Ultraschallprüfung eröffnet neue Möglichkeiten, räumlich ausgedehnte Bauteile mit begrenzter Zugänglichkeit effizient auf ihre Integrität zu prüfen. Die Prüftechnik kann dabei sowohl zur Fehlersuche als auch in einem permanenten Strukturüberwachungssystem und zur Früherkennung von Schäden eingesetzt werden. Jedoch sind bei der Konzeption eines Prüfsystems eine Reihe von Besonderheiten zu beachten:

- Die physikalischen Zusammenhänge, die der Schallausbreitung zu Grunde liegen, sind komplex und erfordern einen höheren Aufwand bei der Signalerzeugung und bei der Auswertung der empfangenen Echosignale verglichen mit konventioneller Ultraschallprüfung.

- Es muss berücksichtigt werden, dass die Wellenausbreitung in verschiedenen Moden stattfindet. Bei der Modenausbreitung tritt - bis auf ganz wenige Ausnahmen - Dispersion auf.

- Für die Erzeugung und Auswertung geführter Wellen ist im Allgemeinen eine mehrkanalige Prüfhardware notwendig. Es reicht nicht, nur die zeitliche Struktur der Echosignale auszuwerten; erst durch die Analyse des Wellenzahlspektrums sind Informationen über die modale Zusammensetzung des Signals auswertbar.

Unter Berücksichtigung der genannten Besonderheiten und Randbedingungen wurden im vorliegenden Beitrag eine Reihe von Prüfsystemen und Anwendungsbeispielen aus dem Bereich der Prüfung plattenförmiger Bauteile und Rohrleitungen vorgestellt, um einen Überblick über die aktuellen Lösungsansätze zu geben. Detailliert wurde auf die Sensortechnik und die Prüfhardware, die für die Konzeption und Entwicklung der Prüftechniken unabdingbar sind, eingegangen. Eine entsprechende Gerätetechnik wird derzeit in der BAM entwickelt und für den Prüfeinsatz validert.

Für die Zukunft wird erwartet, dass die aufgezeigten Lösungsansätze weiter verfeinert werden. Es ist absehbar, dass gerade die Zustandsüberwachung in naher Zukunft stark an Bedeutung gewinnen wird, um die Sicherheit der Bauteile und Komponenten technischer Systeme zu erhöhen und andererseits ihre Lebensdauer möglichst vollständig ausnutzen zu können. Die einfache Verfügbarkeit leistungsfähiger und energiesparend integrierbarer Computerkomponenten wird diesen Trend beschleunigen. Dadurch werden immer komplexere Auswertemechanismen in die Überwachungssysteme implementiert werden können, wie sie für die Ultraschallprüfung mit geführten Wellen erforderlich sind.

\section{Literatur}

[1] J. L. Rose: Ultrasonic Waves in Solid Media. Cambridge University Press, Cambridge 1999.

[2] K.-J. Langenberg, R. Marklein, K. Mayer: Theoretische Grundlagen der zerstörungsfreien Materialprüfung mit Ultraschall. Oldenburg Verlag München, 2009.

[3] P.D. Wilcox, P. Cawley, M.J.S. Lowe: Acoustic fields from PVDF interdigital transducers. IEE Proceedings - Science, Measurement and Technology, Vol. 145(5), 1998. 
[4] J. Prager, C. Hoever: Untersuchungen zur Anregung geführter Wellen in Platten mittels Gruppenstrahlertechnik. 36. Jahrestagung für Akustik, DAGA 2010 (Tagungsband), Düsseldorf, 2010.

[5] J. Prager, C. Hoever, G. Brekow, M. Kreutzbruck: Flaw Detection wit Guided Waves Using Phased Array Technique. 10. ECNDT (Tagungsband), Moskau 2010. 